\title{
Cumplimiento y adherencia a la higiene de manos en trabajadores de la salud de un hospital de Yucatán
}

\section{Compliance and adherence to hand hygiene in health care workers from a hospital in Yucatan}

\author{
Darwin Torres-Erazo*, Mónica E. Sandoval-Balbuena, Nelda J. Núñez-Caamal, \\ Wendy K. Álvarez-Manzanero, Miguel Á. Cicero-Ancona, Milagros B. Carrillo-Basulto, \\ Manuel Narváez-Ricalde y Raquel Chale Dorantes \\ Unidad de Infectología y Vigilancia Epidemiológica, Hospital Regional de Alta Especialidad de la Península de Yucatán, Mérida, Yuc., México
}

\begin{abstract}
Background: Hand hygiene $(\mathrm{HH})$ is effective for infection control, but its performance is poor in health-care workers (HCW). Methods: Study to evaluate the WHO technique and adherence to HH in a third-level hospital in Yucatán, Mexico. Data from evaluation certificates and "shadow" studies were analyzed and expressed in tables and graphs. Results: $406 \mathrm{HCW}$ were evaluated for technique and 141 for adherence. Overall Compliance was $89.2 \%$ (satisfactory), being lower for cleaning (66\%) and inhalation therapy (77\%) teams. The lowest indicator was "hygiene time," with 55.4\% (precarious). Overall Adherence was 61.4\% (minimum), with physicians (12\%) and residents and interns (6\%) being less adherent than nursing staff (69\%). The moments of fewer adherence were "after contact with fluids/secretions" (56.5\%) and "after contact with the patient" (47.6\%). Conclusions: Low adherence at critical moments during patient-care means that HCW did not extend the protection to themselves. Lack of motivation and professional attitude could be the cause of these results.
\end{abstract}

Key words: Compliance. Adherence. Hand hygiene. Five moments. Health-care workers.

\section{RESUMEN}

Introducción: La higiene de manos $(\mathrm{HH})$ es una estrategia eficaz para el control de infecciones, realización es deficiente en trabajadores de la salud (TS). Métodos: Estudio para evaluar el cumplimiento y adherencia a la HH en un hospital de tercer nivel de Yucatán, México. Las cédulas de evaluación y estudios «sombra» fueron analizados y expresados en tablas y gráficos. Resultados: Estudio para evaluar el cumplimiento. Resultados: 406 TS fueron evaluados por técnica y 141 por adherencia. El cumplimiento global fue del 89.2\% (satisfactorio), siendo menor en el área de limpieza (66\%) e inhalo-terapia (77\%). El indicador más bajo $(55.4 \%)$ fue «tiempo de higiene». La adherencia global fue del 61.4\% (mínimo), siendo los médicos (12\%) y residentes/pasantes (6\%) menos adherentes que enfermería (69\%). Los momentos de menor adherencia fueron «después del contacto con fluídos/secreciones» (56.5\%) y "después del contacto con el paciente» (47.6\%). Conclusiones: La baja adherencia en momentos críticos de la atención a pacientes se traduce en que los trabajadores de la salud no extienden la protección hacia ellos mismos. La falta de motivación y la actitud profesional podrían ser la causa de estos resultados.

Palabras clave: Cumplimiento. Adherencia. Higiene de manos. Cinco momentos. Trabajadores de la salud

Correspondence:

*Darwin Torres-Erazo

E-mail: darwintorresera@yahoo.com.mx
Date of reception: 01-06-2020

Date of acceptance: 15-04-2021

DOI: $10.24875 /$ HMCM.20000030
Available online: $25-06-2021$

Hosp Med Clin Manag. 2020;13:154-60

2604-0018 / @ 2021 Mexican Regional Hospitals of High Specialty and Federal Hospitals. Published by Permanyer. This is an open access article under the CC BY-NC-ND license (http://creativecommons.org/licenses/by-nc-nd/4.0/). 


\section{INTRODUCCIÓN}

Las infecciones asociadas a la atención de la salud (IAAS) continúan siendo un importante problema de salud pública ${ }^{1}$, tanto por su relevancia en la seguridad y calidad de atención al paciente ${ }^{2}$ como por el impacto económico que ellas representan para las instituciones de salud alrededor del mundo 3,4 . Esta problemática ha sido tan crucial para la atención sanitaria, que desde 2005 la Alianza Mundial para la Seguridad del Paciente de la Organización Mundial de la Salud (OMS) lanzó la consigna de "Cuidado limpio es cuidado seguro» bajo la premisa de que las IAAS no son intencionales, pero son indeseables e intolerables en el interior de los hospitales ${ }^{5}$.

De acuerdo con la OMS 6 , las IAAS en países desarrollados afectan al $15 \%$ de los pacientes hospitalizados y pueden comprometer hasta al $37 \%$ de los sujetos que ingresan a cuidados intensivos, mientras que en los países en vías de desarrollo las tasas de incidencia y prevalencia de estas infecciones pueden ser hasta 20 veces más altas. En México, para el año 2015 la tasa global de infecciones nosocomiales fue de 4.7 por cada 100 egresos, de acuerdo con lo reportado por la Red Hospitalaria de Vigilancia Epidemiológica ${ }^{7}$, y hasta de del 4.9\% para una institución pública de tercer nivel en Yucatán8.

Ante este enorme desafío planteado por las IAAS, constantemente se han buscado estrategias que permitan controlar, reducir o mitigar las infecciones asociadas a la atención de la salud, pero ninguna ha sido lo suficientemente efectiva como para alcanzar estos objetivos. La OMS ${ }^{6}$ y los Centers for Disease Control and Prevention ${ }^{9}$ consideran que la higiene de las manos es una de las medidas más importantes para prevenir las infecciones intrahospitalarias y la transmisión de microorganismos resistentes dentro de las instituciones. Este sencillo procedimiento es, quizás, la estrategia más costo-efectiva para reducir la transmisión de microorganismos (bacterianos, virales, parasitarios y micóticos $)^{10}$, no solo dentro del hospital, sino también en la comunidad, donde ha permitido reducciones significativas de la carga de enfermedad para otras infecciones prevalentes como la gastroenteritis ${ }^{11}$. Por ello, su recomendación se ha extendido a la salud pública como estrategia de prevención de la transmisión de agentes causantes de epidemias, como ocurrió durante la pandemia de influenza H1N1 de 200912-15, y en
México como una de las acciones esenciales para la seguridad del paciente en los entornos hospitalarios y ambulatorio.

A pesar de la contundente evidencia del beneficio de la higiene de manos, el cumplimiento y apego a los cinco momentos de la OMS son bajos entre los trabajadores de la salud de muchas partes del mundo ${ }^{16}$, por lo que el presente trabajo se propuso conocer los porcentajes de cumplimiento y adherencia a esta estrategia en una institución de salud de tercer nivel de Yucatán (cuya autoevaluación en años previos había alcanzado $>90 \%$ ) y las posibles razones que influyeron en los resultados. Los datos preliminares de este trabajo fueron presentados en el XLIII Congreso Nacional de la Asociación Mexicana de Infectología y Microbiología Clínica, como parte del proceso de comunicación científica de la producción académica realizada en la Unidad de Infectología y Vigilancia Epidemiológica (UIVEH) del Hospital Regional de Alta Especialidad de la Península de Yucatán (HRAEPY).

\section{MÉTODOS}

Se realizó un estudio retrospectivo, observacional y descriptivo para evaluar el cumplimiento de la técnica y la adherencia a los cinco momentos de la higiene de manos de la OMS, en el HRAEPY, una institución pública ubicada en el sureste mexicano que cuenta con aproximadamente 1,000 trabajadores de la salud, incluyendo médicos, paramédicos, becarios, administrativos y personal de apoyo logístico.

Todas las centrales de enfermería del hospital, las habitaciones de los pacientes y varias áreas administrativas tienen ayudas visuales de cómo hacer la higiene de manos, un lavamanos con agua, toallas de papel, dispensador de jabón disponible durante todo el año y, en las áreas donde no es posible tener estos recursos, existe al menos un dispensador de alcohol-gel. Adicionalmente, existe un programa anual calendarizado de capacitación en higiene de manos para el personal de base (dos veces al año) y para el personal rotativo de nuevo ingreso (siete veces al año).

La información para este estudio se obtuvo a partir de los registros de las cédulas de evaluación elaboradas por la UIVEH basadas en los lineamientos de la OMS ${ }^{6}$ para evaluar el conocimiento de los 17 pasos que seguir 
para la correcta higiene de manos en una solicitud proactiva por parte del personal de la unidad y de los «estudios sombra» realizados por la UIVEH durante 2017, definiéndose como "estudio sombra» al procedimiento mimetizado de observación para evaluar visualmente a una persona (inconsciente de que está siendo observada) en el cumplimiento y adherencia a los cinco momentos de la higiene de manos recomendados por la OMS que corresponden a: «antes de entrar en contacto con el paciente», "antes de realizar un procedimiento aséptico o manipular dispositivos en el paciente", «después del contacto con fluidos y secreciones del paciente», "después del contacto directo con el paciente» $y$ «después de salir del entorno/cosas del paciente».

Los registros de las cédulas de evaluación y estudios sombra fueron realizados en forma aleatorizada, en diversas áreas del hospital y sin discriminación del grado académico o de posición laboral dentro de la institución. La información registrada se recolectó en una base de datos de Excel cuyos resultados se presentan mediante estadística descriptiva en tablas y gráficos, expresados en porcentajes a partir de una estrategia de «semaforización», entendiéndose como tal la asignación de un color simbólico a cada resultado porcentual, que para el caso de este estudio tuvo el siguiente significado: negro $=$ precario $(<60 \%)$, rojo $=$ mínimo (60 a $<80 \%)$, amarillo = satisfactorio $(80 \mathrm{a}<90 \%)$ y verde $=$ sobresaliente $(\geq 90 \%)$.

\section{RESULTADOS}

De enero a diciembre de 2017 se evaluaron 547 trabajadores de la salud del HRAEPY para determinar el nivel de cumplimiento (conocimiento) de la técnica y el grado de adherencia a los cinco momentos de la higiene de manos recomendado por la OMS.

Del total de sujetos incluidos, 406 personas fueron evaluadas para determinar el cumplimiento de la técnica y 141 fueron evaluadas mediante "estudio sombra" para conocer el grado de apego/adherencia a este procedimiento.

El porcentaje global de cumplimiento (derivado del conocimiento) de la técnica del lavado de manos fue del 89.2\% (equivalente a satisfactorio). En la distribución categórica, el mayor número de evaluados por técnica fueron personal de enfermería $(n=195,48 \%)$, seguidos de médicos $(n=118,29 \%)$, químicos $(n=24,6 \%)$ y «otros», que incluyeron personal administrativo no médico, camilleros, personal de intendencia, técnicos de inhaloterapia, etc. $(n=69,17 \%)$.

Los trabajadores/áreas con mayor cumplimiento en forma global (> 90\%) fueron: enfermería, médicos de base, personal de laboratorio, trabajo social y hemodiálisis, mientras que los servicios con menor cumplimiento fueron limpieza e inhaloterapia (66 y $77 \%$, respectivamente). El personal becario (residentes, internos y médicos pasantes de servicio social) tuvo un porcentaje de cumplimiento intermedio que estuvo entre el 85 y el $89 \%$.

El análisis de cumplimiento de los 17 pasos que seguir en la higiene de manos mostró que uno de los pasos con menor porcentaje de cumplimiento durante todos los meses, y en general a lo largo de todo el año, fue el tiempo empleado en el procedimiento («termina el procedimiento en un tiempo igual o mayor a 60 segundos»), con el $55.4 \%$ (equivalente a precario). Los porcentajes y equivalencias de los diferentes pasos evaluados a lo largo del 2017 se muestran en la figura 1.

En la evaluación del apego a los cinco momentos de la higiene de manos, los "estudios sombra" revelaron que de forma global el personal del HRAEPY estaba adherido a estos momentos en el $61.4 \%$ (equivalente a mínimo) y los momentos con menores resultados de adherencia/apego fueron "después del contacto con fluidos/secreciones» y "después del contacto con el paciente» (56.5 y $47.6 \%$, respectivamente) (Fig. 2). El personal sanitario con menor desempeño de adherencia a estos dos momentos fue: técnicos en rayos $X$ y estudiantes de medicina ( $0 \%$ en ambos momentos), médicos internos de pregrado y residentes de posgrado con $0 \%$ de apego al lavado de manos «después del contacto con fluidos y secreciones».

En el análisis categórico y comparativo para la adherencia a los cinco momentos de la higiene de manos, los resultados mostraron que el personal de electrodiagnóstico, junto con trabajo social, son las áreas con mejor calificación (80 y 70\%, respectivamente), mientras que los estudiantes de medicina y el personal técnico de rayos $X$ tuvieron los peores desempeños (40 y $20 \%$, respectivamente). El personal becario (internos de pregrado, residentes), médicos de base, enfermería, camilleros y personal de laboratorio tuvieron diferentes porcentajes de cumplimiento, cuyos resultados se muestran en la figura 3. 


\begin{tabular}{|c|c|c|c|c|c|c|c|c|c|c|c|c|c|}
\hline Acciones & Enero & Febrero & Marzo & Abril & Mayo & Junio & Julio & Agosto & Septiembre & Octubre & Noviembre & Diciembre & 2017 \\
\hline $\begin{array}{l}\text { Se retira anillos, pulseras, relojes y } \\
\text { otros objetos de las manos previo al } \\
\text { lavado }\end{array}$ & 89.47 & 85.71 & 77.78 & 75 & 82.69 & 52.38 & 82.80 & 100 & 94.87 & 93.33 & 73.33 & 87.5 & 82.91 \\
\hline Abre la llave y se moja las manos & 90.79 & 82.86 & 88.89 & 100 & 84.62 & 100 & 86.02 & 100.00 & 100.00 & 100.00 & 100.00 & 93.75 & 93.91 \\
\hline $\begin{array}{l}\text { Deposita el jabón en la palma de una } \\
\text { mano en forma suficiente }\end{array}$ & 92.11 & 85.71 & 100 & 100 & 86.54 & 100 & 87.10 & 100.00 & 100.00 & 100.00 & 100 & 93.75 & 95.43 \\
\hline 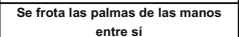 & 93.42 & 91.43 & 88.89 & 81.25 & 92.31 & 100 & 91.40 & 100.00 & 100.00 & 93.33 & 100.00 & 100 & 94.34 \\
\hline $\begin{array}{l}\text { Se frota la palma de la mano } \\
\text { derecha contra el dorso de la mano } \\
\text { izquierda }\end{array}$ & 89.47 & 88.57 & 81.48 & 87.5 & 92.31 & 95.24 & 88.17 & 94.44 & 94.87 & 93.33 & 80.00 & 100 & 90.45 \\
\hline $\begin{array}{l}\text { Se frota la palma de la mano } \\
\text { izquierda contra el dorso de la mano } \\
\text { derecha }\end{array}$ & 88.16 & 88.57 & 81.48 & 100 & 92.31 & 95.24 & 88.17 & 88.89 & 97.44 & 100 & 100 & 100 & 93.35 \\
\hline $\begin{array}{l}\text { Se frota la palma de las manos entre } \\
\text { si entrelazando los dedos }\end{array}$ & 77.63 & 74.29 & 81.48 & 87.5 & 82.69 & 100 & 69.89 & 94.44 & 94.87 & 93.33 & 100 & 87.5 & 86.97 \\
\hline $\begin{array}{l}\text { Se frota el dorso de los dedos de la } \\
\text { mano derecha contra la palma de la } \\
\text { mano izquierda }\end{array}$ & 88.16 & 74.29 & 88.89 & 68.75 & 90.38 & 85.71 & 82.80 & 88.89 & 87.18 & 100 & 100 & 100 & 87.92 \\
\hline $\begin{array}{l}\text { Se frota el dorso de los dedos de la } \\
\text { mano izquierda contra la palma de la } \\
\text { mano derecha }\end{array}$ & 88.16 & 74.29 & 88.89 & 81.25 & 90.38 & 80.95 & 83.87 & 94.44 & 79.49 & 100 & 100 & 100 & 88.48 \\
\hline $\begin{array}{l}\text { Atrapa con la palma de la mano } \\
\text { derecha el dedo pulgar de la mano } \\
\text { izquierda y lo frota }\end{array}$ & 90.79 & 71.43 & 81.48 & 81.25 & 88.46 & 80.95 & 91.40 & 94.44 & 89.74 & 100 & 100 & 100 & 89.16 \\
\hline $\begin{array}{l}\text { Atrapa con la palma de la mano } \\
\text { izquierda el dedo pulgar de la mano } \\
\text { derecha y lo frota }\end{array}$ & 90.79 & 77.14 & 77.78 & 87.5 & 88.46 & 76.19 & 90.32 & 88.89 & 92.31 & 100 & 93.33 & 100 & 88.56 \\
\hline $\begin{array}{l}\text { Se frota la punta de los dedos de la } \\
\text { mano derecha contra la palma de la } \\
\text { mano izquierda }\end{array}$ & 89.47 & 82.86 & 92.59 & 93.75 & 88.46 & 90.48 & 84.95 & 88.89 & 94.87 & 100 & 100 & 100 & 92.19 \\
\hline $\begin{array}{c}\text { Se frota la punta de los dedos de la } \\
\text { mano izquierda contra la palma de la } \\
\text { mano derecha }\end{array}$ & 88.16 & 82.86 & 92.59 & 93.75 & 84.62 & 90.48 & 83.87 & 83.33 & 94.87 & 100 & 100 & 100 & 91.21 \\
\hline Se enjuaga las manos con agua & 96.05 & 94.29 & 96.30 & 87.5 & 94.23 & 100 & 89.25 & 88.89 & 100 & 100 & 100 & 100 & 95.54 \\
\hline $\begin{array}{l}\text { Se seca las manos con papel } \\
\text { deshechable }\end{array}$ & 94.74 & 94.29 & 96.30 & 100 & 90.38 & 100 & 93.55 & 88.89 & 97.44 & 100 & 100 & 100 & 96.30 \\
\hline $\begin{array}{l}\text { Cierra la llave cubriéndola con el } \\
\text { mismo papel desechable }\end{array}$ & 92.11 & 94.29 & 96.30 & 100 & 88.46 & 100 & 83.87 & 88.89 & 97.44 & 100 & 93.33 & 100 & 94.56 \\
\hline $\begin{array}{l}\text { Termina el procedimiento en un } \\
\text { tiempo mayor a } 60 \text { segundos }\end{array}$ & 64.47 & 62.86 & 59.26 & 93.75 & 53.85 & 76.19 & 53.76 & 50 & 48.72 & 6.67 & 26.67 & 68.75 & 55.41 \\
\hline
\end{tabular}

\begin{tabular}{|c|c|}
\hline \multicolumn{2}{|c|}{ Semáforo de cumplimiento } \\
\hline Interpretación & $\begin{array}{c}\text { Porcentaje } \\
\%\end{array}$ \\
\hline Precario & $<60$ \\
\hline Mínimo & $60 \mathrm{a}<80$ \\
\hline Satisfactorio & $80 \mathrm{a}<90$ \\
\hline Sobresaliente & $\geq 90$ \\
\hline
\end{tabular}

Figura 1. Porcentajes globales (por mes y año) del cumplimiento de la técnica de lavado de manos de la Organización Mundial de la Salud, en trabajadores de la salud del Hospital Regional de Alta Especialidad de la Península de Yucatán de acuerdo con la estrategia de semaforización.

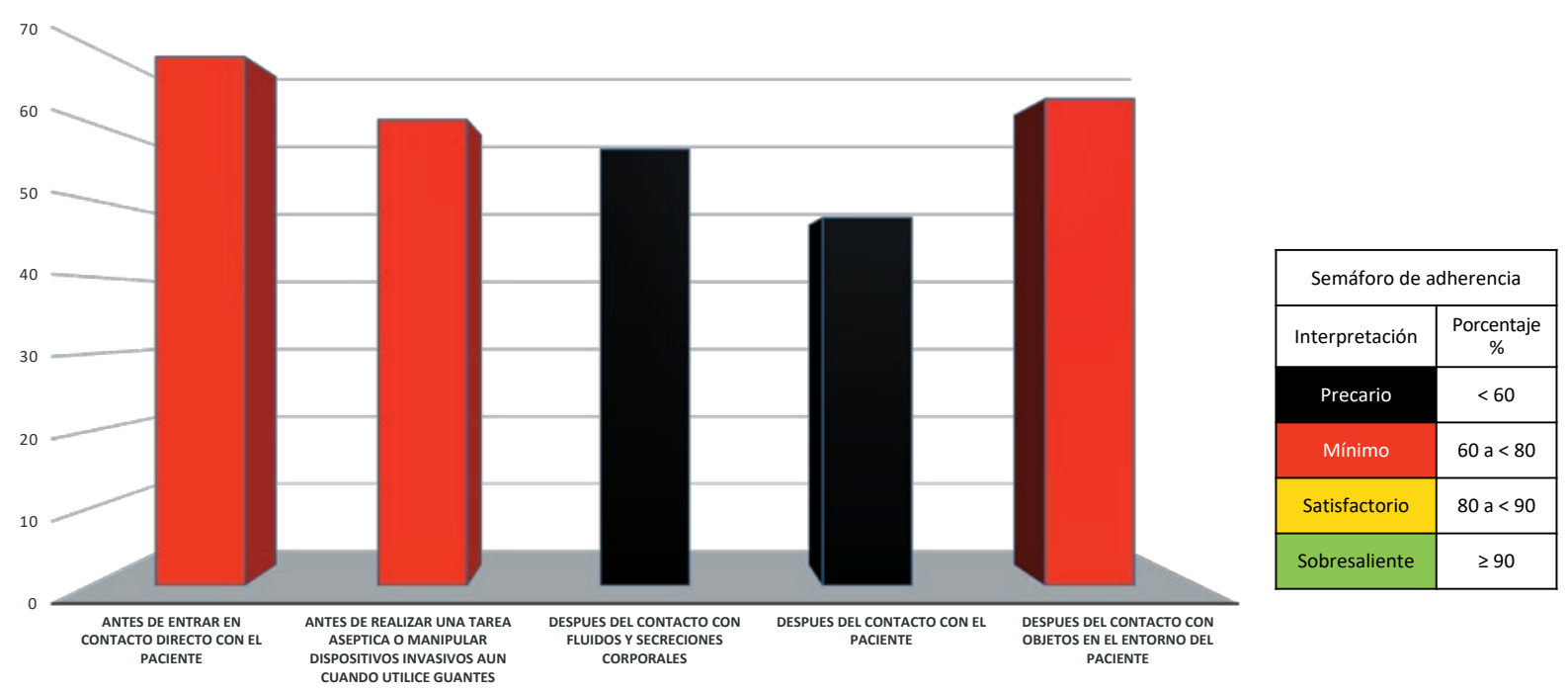

Figura 2. Porcentajes de adherencia a los cinco momentos del lavado de manos de la Organización Mundial de la Salud en los trabajadores de la salud del Hospital Regional de Alta Especialidad de la Península de Yucatán durante 2017.

\section{DISCUSIÓN}

Los resultados de este estudio mostraron que los porcentajes de cumplimiento y adherencia a la higiene de manos son variables para cada uno de los pasos de la técnica y entre las diferentes categorías evaluadas.
El porcentaje global de cumplimiento para el HRAEPY fue satisfactorio (89.2\%) en comparación con otros autores que han demostrado porcentajes menores al 50\% en hospitales similares de África y el Caribe ${ }^{17,18}$. Este resultado traduce que la ejecución del programa anual de capacitación en higiene de manos establecido por la UIVEH para todo el personal que labora en la institución 


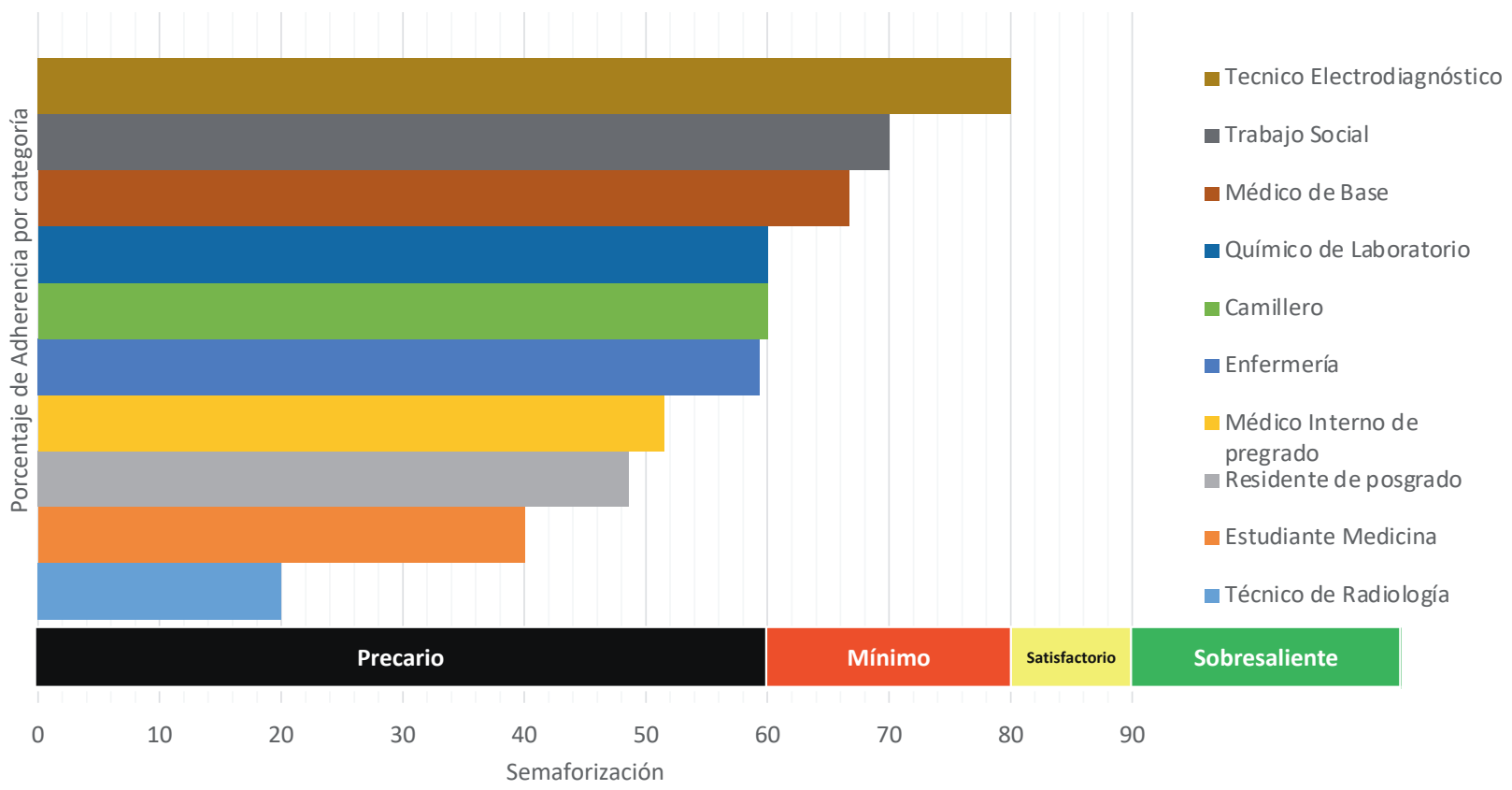

Figura 3. Distribución porcentual de adherencia a los cinco momentos de la Organización Mundial de la Salud por categoría en el Hospital Regional de Alta Especialidad de la Península de Yucatán.

y al personal de nuevo ingreso rinde resultados aceptables para el aprendizaje y conocimiento de esta estrategia de calidad en la atención a pacientes hospitalizados, lo cual coincide con los hallazgos de otros autores ${ }^{19,20}$.

A pesar de este buen resultado, hubo un indicador con muy bajo porcentaje de cumplimiento (precario), que correspondió al tiempo utilizado en la higiene de manos. Más de la mitad de los evaluados (55.4\%) terminó el procedimiento en menos de 60 segundos (tiempo recomendado para que sea eficaz), y la justificación para este incumplimiento fue atribuida a la «sobrecarga laboral» y a la necesidad de «no perder tiempo» en un procedimiento que, según su percepción, «afecta a su rutina de trabajo". Este resultado es similar al de otro autor ${ }^{21}$ que también demostró tiempos menores a los recomendados para la higiene de manos, pero encontró diferencias al comparar personal médico vs. enfermeras y entre las áreas clínicas vs. quirúrgicas o de cuidado intensivo. Estos hallazgos contrastan con nuestro trabajo, ya que en el HRAEPY el personal de enfermería tiene mayor demanda laboral por turno, pero no por ello menor cumplimiento o adherencia a la higiene de manos en comparación con personal médico (residentes, médicos de base y becarios de pregrado). Este hallazgo deja sin justificación el argumento de que la «sobrecarga laboral» impide el cumplimiento correcto de la higiene de manos (en técnica y tiempo), puesto que el personal de enfermería realiza más actividades asistenciales y en consecuencia tiene mayor número de oportunidades en las cuales debe lavarse las manos ${ }^{22}$.

Debido a que esta diferencia ya había sido evidenciada desde años previos, en 2015 se estableció en el HRAEPY la recomendación basada en «modelos que seguir» propuesta por Lankford ${ }^{23}$, cuyo propósito es la promoción/reconocimiento del personal médico o de enfermería que «cumple con la higiene de manos de manera ejemplar» para reforzar el hábito de este procedimiento, ya que se ha observado que la conducta de los profesionales influye positivamente sobre sus colegas u otros trabajadores de la salud y favorece que los estudiantes imiten la conducta de higiene de manos de sus superiores o que la incumplan cuando ellos no la ejercen ${ }^{24}$. Esta estrategia no pudo mantenerse en el hospital debido a diversas causas y no mostró los resultados beneficiosos encontrados por el autor de la propuesta al implementarla como medida de mitigación conductual frente al incumplimiento o desapego a la higiene de manos.

Otro de los hallazgos de interés epidemiológico, y probablemente muy relacionado con la seguridad laboral en este estudio, fue que el personal del HRAEPY está 
mínimamente adherido de forma global (61.4\%) a los cinco momentos de la higiene de manos recomendada por la OMS. Esto fue más evidente en el personal médico (de base y becario) que omite la higiene de manos en dos de los momentos más críticos para la transmisión de microorganismos, no solo hacia otros pacientes, sino hacia ellos mismos, lo cual representa un significativo riesgo para la transmisión nosocomial de enfermedades infecciosas desde los pacientes hacia el personal de salud y entre los pacientes en el interior del hospital.

Este hallazgo difiere de lo reportado en otras publicaciones, donde el personal sanitario es más proclive a la higiene de manos precisamente después del contacto con el paciente ${ }^{18}$ y más aún después del contacto con fluidos o secreciones ${ }^{17}$. Esta condición requirió de atención urgente en el HRAEPY por parte de las diversas áreas involucradas en la concienciación de esta necesidad y fueron corregidas inmediatamente al ser identificadas, por medio de retroalimentación proactiva hacia el personal que presentó esta omisión durante los estudios sombra y en las capacitaciones subsecuentes.

La justificación para estos contrastantes resultados, en los que el personal sanitario conoce muy bien la técnica, pero no aplica los conocimientos adquiridos para su trabajo asistencial diario, probablemente tenga una explicación conductual, relacionada con la carencia de competencias profesionales/académicas o simplemente a "creencias", como han planteado algunos autores $^{24-28}$. En una revisión sistemática reciente, los factores de conducta que se han identificado entre los trabajadores de la salud que no se adhieren o no cumplen el lavado de manos fueron: malos hábitos adquiridos y sostenidos durante el entrenamiento médico, carencia de competencias académicas adquiridas en la universidad, ausencia de ejemplos positivos y motivación por parte de sus superiores, temor o vergüenza a ser observados/criticados si realizan la higiene de manos frente a sus colegas, ausencia de actitudes proactivas o positivas frente a las indicaciones o solicitudes de hacer higiene de manos o simplemente el desinterés por cumplir con el procedimiento debido a la ignorancia del impacto de esta estrategia sobre las IAAS ${ }^{29}$.

Las fortalezas de este estudio incluyeron el tamaño de la muestra (aproximadamente el $50 \%$ del personal hospitalario) y el haber evaluado conjuntamente el conocimiento de la técnica para contrastarlo con la adherencia y la inclusión de "estudios sombra» que, al ser ciegos para los participantes (porque no son conscientes de que están siendo observados), permiten resultados más objetivos, como lo señala la OMS. Las limitaciones se relacionan con las dificultades para evaluar al personal en otros horarios, como el nocturno o vespertino, y la ausencia de medición de la influencia de estos hallazgos sobre la frecuencia y prevalencia de las IAAS en el HRAEPY, como se realizó en otro estudio plasmado por la UIVEH al evaluar el impacto de un programa de gerencia de antimicrobianos sobre las IAAS ${ }^{30}$.

\section{CONCLUSIONES}

A pesar de que la higiene de manos es un procedimiento sencillo y nada complejo, el cumplimiento y adherencia pueden tener diferencias sustanciales en una misma institución. La evaluación de la higiene de manos en el HRAEPY tuvo resultados satisfactorios debido a que el personal conoce y ejecuta de manera apropiada la técnica de este procedimiento, (aunque no lo haga dentro del tiempo apropiado), pero se evidenció que el personal médico no extiende el cuidado y protección hacia ellos mismos o hacia los pacientes, y no hay una explicación evidente para esta conducta. Es probable que la falta de motivación y la actitud profesional sean los determinantes para la omisión de este sencillo procedimiento, que es considerado como un indicador de la calidad y seguridad en la atención intrahospitalaria, con un enorme impacto a nivel poblacional-comunitario cuando se trata de controlar infecciones. Los resultados de este trabajo son el preámbulo de estudios futuros para evaluar y plantear estrategias adicionales que permitan mejorar los porcentajes de adherencia y reducir las tasas de las IAAS en el HRAEPY, particularmente en el contexto de que se trata de un hospital que forma a personal becario de medicina (internos, residentes y subespecialistas), en quienes es mandatorio mantener vigente el ejercicio de esta actividad, como herramienta de mitigación de las infecciones asociadas a los cuidados de la salud.

\section{FINANCIAMIENTO}

Los autores no recibieron patrocinio para llevar a cabo este artículo. 


\section{CONFLICTO DE INTERESES}

Los autores declaran no tener ningún conflicto de intereses.

\section{RESPONSABILIDADES ÉTICAS}

Protección de personas y animales. Los autores declaran que para esta investigación no se han realizado experimentos en seres humanos ni en animales.

Confidencialidad de los datos. Los autores declaran que en este artículo no aparecen datos de pacientes.

Derecho a la privacidad y consentimiento informado. Los autores declaran que en este artículo no aparecen datos de pacientes.

\section{BIBLIOGRAFÍA}

1. Al-Tawfiq JA, Tambyah PA. Healthcare associated infections (HAI) perspectives. J Infect Pub Health. 2014;7:339-44.

2. Sreeramoju P, Dura L, Fernandez ME, Minhajuddin A, Simacek K, Fomby TB, et al. Using a positive deviance approach to influence the culture of patient safety related to infection prevention. Open Forum Infect Dis. 2018:5(10):1-8

3. Zimlichman E, Henderson D, Tamir 0, Franz C, Song P, Yamin CK, et al. Health care-associated infections: a meta-analysis of costs and financial impact on the US health care system. JAMA Intern Med. 2013;173: 2039-46.

4. Umscheid CA, Mitchell MD, Doshi JA, Agarwal R, Williams K, Brennan PJ. Estimating the proportion of healthcare-associated infections that are reasonably preventable and the related mortality and costs. Infect Control Hosp Epidemiol. 2011;32(2):101-14.

5. Pittet D, Donaldson L. Clean care is safer care. Lancet. 2006;366(8): 1246-7.

6. World Health Organization. WHO guidelines on hand hygiene in health care [Internet]. World Health Organization; 2009. Disponible en: https://www. who.int/gpsc/5may/tools/who guidelines-handhygiene summary.pdf

7. Red Hospitalaria de Vigilancia Epidemiológica, Informe Anual 2015 [Internet]. México: Dirección General de Epidemiología, Secretaría de Salud; 2016. Disponible en: https://www.gob.mx/cms/uploads/attachment/ file/212974/infoanual rhove_2015.pdf

8. Torres-Erazo D, Domínguez-Méndez J, Buenfil-Vera L, Cicero-Ancona M. Características clínicas y microbiológicas de pacientes con infecciones asociadas a los cuidados de la salud en un hospital de alta especialidad de Yucatán. Enf Inf Microbiol. 2018:38(2):44-9.

9. Boyce JM, Pittet D; Healthcare Infection Control Practices Advisory Committee; HICPAC/SHEA/APIC/IDSA Hand Hygiene Task Force. Centers for Disease Control and Prevention. Guideline for Hand Hygiene in Health-Care Settings: Recommendations of the Healthcare Infection Control Practices Advisory Committee and the HICPAC/SHEA/APIC/IDSA Hand Hygiene Task Force. Society for Healthcare Epidemiology of America/Association for Professionals in Infection Control/Infectious Diseases Society of America. MMWR Recomm Rep. 2002;51(RR-16):1-45, quiz CE1-4.
10. Pittet D, Allegranzi B, Sax H, Dharon S, Pessoa-Silva CL, Donaldson L, et al. Evidence-based model for hand transmission during patient care and the role of improved practices. Lancet Infect Dis. 2006;6:641-52.

11. Pittet D. Clean hands reduce the burden of disease. Lancet 2005;366(16):185-7

12. World Health Organization. Pandemic influenza preparedness and response: WHO guidance document [Internet]. World Health Organization; 2009. Disponible en: https://www.who.int/influenza/resources/documents/pandemic_guidance_04_2009/en/

13. Moncion K, Young K, Tunis M, Rempel S, Stirling R, Zhao L. Effectiveness of hand hygiene practices in preventing influenza virus infection in the community setting: A systematic review. Can Commun Dis Rep. 2019;45(1):12-23

14. Warren-Gash C, Fragaszy E, Hayward AC. Hand hygiene to reduce community transmission of influenza and acute respiratory tract infection: a systematic review. Influenza Other Resp Vir. 2012;7(5):738-49.

15. Bin Abdulrahman AK, Bin Abdulrahman KA, Almadi MK, Alharbi AM, Mahmoud MA, Almasri MS, et al. Do various personal hygiene habits protect us against influenza-like illness? BMC Pub Health. 2019;19:1324.

16. Pittet D, Allegranzi B, Boyce J, World Health Organization World Alliance for Patient Safety First Global Patient Safety Challenge Core Group of Experts. The World Health Organization Guidelines on Hand Hygiene in Health Care and Their Consensus Recommendations. Infect Control Hosp Epidemiol. 2009;30(7):611-22.

17. Kokola T, Gezahegn T. A twenty-four-hour observational study of hand hygiene compliance among healthcare workers in Debre Berhan referral hospital, Ethiopia. Antimicrob Res Infect Control. 2017:6:109.

18. Nicholson AM, Tennant IA, Martin AC, Ehikhametalor K, Reynolds G, Thoms-Rodriguez CA, et al. Hand hygiene compliance by health care workers at a teaching hospital, Kingston, Jamaica. J Infect Dev Ctries. 2016;10(10):1088-92.

19. Moghaddam PS, Arjmandnia M, Shokrollahi M, Aghaali M. Does training improve compliance with hand hygiene and decrease infections in the neonatal intensive care unit? A prospective Study. J Neonatal-Perinatal Med. 2015;8(3):221-5.

20. Pittet D, Hugonnet S, Harbarth S, Mourouga P, Sauvan V, Touveneau S, et al. Effectiveness of a hospital-wide programme to improve compliance with hand hygiene. Infection Control Programme. Lancet. 2000:356(9238):1307-12.

21. Stahmeyer JT, Lutze B, von Lengerke T, Chaberny IF, Krauth C. Hand hygiene in intensive care units: A matter of time? J Hosp Infect. 2017;95(4):338-43.

22. Syed A, Juergens C, McLaws ML. An average hand hygiene day for nurses and physicians: The burden is not equal. Am J Infect Control. 2016; 44(7):777-81.

23. Lankford MG, Zembower TR, Trick WE, Hacek DM, Noskin GA, Peterson LR. Influence of role models and hospital design on hand hygiene of healthcare workers. Emerg Infect Dis. 2003;9:217-23.

24. Erasmus V, Brouwer W, van Beeck EF, Oenema A, Daha TJ, Richardus JH, et al. A qualitative exploration of reasons for poor hand hygiene among hospital workers: Lack of positive role models and of convincing evidence that hand hygiene prevents cross-infection. Infect Control Hosp Epidemiol. 2009;30:415-9.

25. Gartmeier M, Baumgartner M, Burgkart R, Heiniger S, Berberat PO. Why hand hygiene is not sufficient: modeling hygiene competence of clinical staff as a basis for its development and assessment. GMS J Med Edu. 2019;36(4):1-21.

26. Whitby M, McLaws ML, Ross M. Healthcare workers don't wash their hands: A behavioral explanation. Infect Control Hosp Epidemiol. 2006:27:484-92.

27. Ibrahim MAB, Chow C, Poh BF, Ang B, Chow A. Differences in psychosocial determinants of hand hygiene between health care professional groups: Insights from a mixed-methods analysis. Am J Infect Control. 2017; 46(3):253-60.

28. Pittet D, Simon A, Hugonnet S, Pessoa-Silva CL, Sauvan V, Perneger TV. Hand hygiene among physicians: performance, beliefs, and perceptions. Ann Intern Med 2004; 141:1-8.

29. Seo HJ, Sohng KY, Chang SO, Chaung SK, Won JS, Choi MJ. Interventions to improve hand hygiene compliance in emergency departments: a systematic review. J Hosp Infect. 2019:102:394-406.

30. Torres-Erazo DS, Nuñez-Caamal NJ, Carrillo-Basulto MB, Cicero-Ancona M, Cuevas-Sosa LA, Escuadrón meropenem. Impact of an antimicrobia stewardship program on the healthcare-associated infections in a third-leve hospital in Yucatán, Mexico. Open Forum Infect Dis. 2019;6(Suppl.2):S687. 